Bicocca-FT-00-01

IFUP-TH 2000-10

\title{
Topological susceptibility in full QCD at zero and finite temperature
}

\author{
B. Allés ${ }^{\mathrm{a}}$, M. D'Elia ${ }^{\mathrm{b}}$ and A. Di Giacomo ${ }^{\mathrm{b}}$ \\ ${ }^{a}$ Dipartimento di Fisica, Università di Milano-Bicocca and \\ INFN-Sezione di Milano, I-20133 Milano, Italy \\ b Dipartimento di Fisica, Università di Pisa and INFN-Sezione di Pisa, I-56127 Pisa, Italy
}

\begin{abstract}
We present a study of the topological susceptibility $\chi$ on the lattice for full QCD with 2 and 4 flavours of staggered fermions at zero and finite temperature $T$. We find that $\chi$ presents a sharp drop across the deconfinement transition. We also study the dependence of $\chi$ on the quark mass at $T=0$ : we have no conclusive evidence for the expected chiral behaviour.
\end{abstract}




\section{Introduction}

The topological susceptibility $\chi$ plays a relevant role in understanding several low energy properties of QCD. It is defined as the zero-momentum two-point correlation function of the topological charge density $Q(x)$ [1], 国],

$$
\begin{aligned}
\chi & \equiv \int \mathrm{d}^{4} x \partial_{\mu}\left\langle 0\left|\mathrm{~T}\left\{K^{\mu}(x) Q(0)\right\}\right| 0\right\rangle, \\
Q(x) & \equiv \frac{g^{2}}{64 \pi^{2}} \epsilon^{\mu \nu \rho \sigma} F_{\mu \nu}^{a}(x) F_{\rho \sigma}^{a}(x),
\end{aligned}
$$

where $K^{\mu}(x)$ is the Chern current

$$
K^{\mu}(x) \equiv \frac{g^{2}}{16 \pi^{2}} \epsilon^{\mu \nu \rho \sigma} A_{\nu}^{a}(x)\left(\partial_{\rho} A_{\sigma}^{a}(x)-\frac{1}{3} g f^{a b c} A_{\rho}^{b}(x) A_{\sigma}^{c}(x)\right),
$$

which satisfies $Q(x)=\partial_{\mu} K^{\mu}(x)$. In these expressions $g$ is the QCD coupling constant and $f^{a b c}$ are the structure functions of SU(3). In Eq. (四) we keep the derivative out of the expectation value in the definition of $\chi$ according to the continuum prescription [3].

The topological susceptibility in the quenched theory at zero temperature has been widely studied. In particular, a non-zero value of $\chi$ provides an explanation for the large mass of the $\eta^{\prime}$ particle, the would-be Goldstone boson of the axial symmetry [1]. In Ref. [4] we have found that the value of $\chi$ in pure Yang-Mills theory is $\chi=(175(5) \mathrm{MeV})^{4}$ in agreement with the phenomenological expectation [2]. For a review of other calculations see [5] and references therein.

In full QCD, Ward identities and current algebra relations imply in the chiral limit [2, 6]

$$
\chi=\frac{m}{N_{f}}\langle\bar{\psi} \psi\rangle
$$

where $m$ is the fermion mass and $N_{f}$ the number of fermions. It is interesting to verify on the lattice the linear dependence on the mass displayed by Eq. (3).

The topological susceptibility at non-zero temperature for pure Yang-Mills theory has also been studied. In Ref. [4] it has been shown that it is rather constant versus $T$ below the deconfinement temperature $T_{c}$ and it undergoes a sharp drop across the phase transition. It is also interesting to study the behaviour of $\chi$ for full QCD as a function of $T$. In particular, Eq. (3) suggests the existence of a drop in the signal of $\chi$ across $T_{c}$ also for the full QCD case [6], (see also [7]).

In the present paper we will present a lattice determination of $\chi$ in full QCD for $N_{f}=2$ degenerate flavours of staggered fermions at finite and zero temperature and for $N_{f}=4$ at finite temperature. The Monte Carlo simulation was done on the APE-Quadrics in Milano and Pisa.

The method used to extract $\chi$ from the lattice is described in Section 2. The results and technical details of the simulation for the $N_{f}=4$ and $N_{f}=2$ cases are shown in Section 3 and Section 1 respectively. The conclusions are drawn in the last section. 


\section{Method to determine $\chi$}

We have simulated the theory on a space-time lattice. The topological charge density has been measured by making use of the lattice operator

$$
Q_{L}(x) \equiv-\frac{1}{2^{9} \pi^{2}} \sum_{\mu \nu \rho \sigma= \pm 1}^{ \pm 4} \tilde{\epsilon}_{\mu \nu \rho \sigma} \operatorname{Tr}\left(\Pi_{\mu \nu}(x) \Pi_{\rho \sigma}(x)\right)
$$

where $\Pi_{\mu \nu}(x)$ is a plaquette in the $\mu-\nu$ plane and $\widetilde{\epsilon}_{\mu \nu \rho \sigma}$ is a generalized Levi-Civita tensor which acquires an extra minus sign for each of its indices going negative. Green functions containing $Q_{L}(x)$ differ from the continuum counterparts by a finite renormalization $Z$ [ 8 ], in short

$$
Q_{L}(x)=Z a^{4} Q(x)+O\left(a^{6}\right),
$$

where $a$ is the lattice spacing. Actually $Q_{L}(x)$ mixes with fermionic operators during renormalization but it has been shown that the off-diagonal mixings are negligible [9].

The lattice topological susceptibility $\chi_{L}$ is defined as

$$
\chi_{L} \equiv \frac{\left\langle Q_{L}^{2}\right\rangle}{V}
$$

where $V$ is the lattice space-time volume and $Q_{L}$ is the total topological charge, $Q_{L} \equiv$ $\sum_{x} Q_{L}(x)$. The definition of $\chi_{L}$ differs from the continuum expression for $\chi$ in Eq. (11) by contact terms which must be subtracted. The relationship between $\chi$ and $\chi_{L}$ is

$$
\chi_{L}=a^{4} Z^{2} \chi+M
$$

where $M$ contains mixings with operators with compatible quantum numbers.

We have measured $Q_{L}(x)$ after having applied two smearing steps with smearing parameter $c=0.9$ [10]. The smearing process allows the operator to be less sensitive to the quantum fluctuations. Therefore the renormalization constant $Z$ approaches 1 and $M$ approaches 0 . As a consequence the statistical errors are strongly diminished [10].

We evaluate $Z$ and $M$ by using the "heating method" which provides a non-perturbative determination of the renormalization constants [11]. To calculate $Z$, several steps of an updating algorithm (the same used during the Monte Carlo simulation) are applied on a configuration containing a charge +1 classical instanton. These updatings thermalize first the quantum fluctuations, responsible for the renormalization effects, and due to the slowing down leave the topological content unchanged. The measurement of $Q_{L}$ on such updated configurations yields $Z \cdot Q$. As $Q$ is known, one can extract $Z$. Notice that this procedure is equivalent to imposing the continuum value for the 1 -instanton charge (in the $\overline{\mathrm{MS}}$ scheme it is +1 ) and extracting the finite multiplicative renormalization $Z$ by evaluating a matrix element of $Q_{L}$.

The additive renormalization $M$ is obtained in a similar way. We apply a few heating

steps with the same updating algorithm as above on a zero-field configuration. The 
topological susceptibility is then measured. This provides the value of $M$, if no instantons have been created during the few updating steps. As explained in [4], cooling tests must be done to check that the background topological charge has not been changed during the heating. Configurations where the topological content has been changed are discarded from the sample.

Once $Z$ and $M$ are known, we can extract $a^{4} \chi$ from Eq. (7). We have fixed $a$ in physical units by measuring the string tension $\sigma$ on a $16^{4}$ lattice and assuming that $\sqrt{\sigma}=420 \mathrm{MeV}$. To this purpose, Wilson loops ranging from $1 \times 1$ to $8 \times 8$ have been evaluated by using smeared spatial links and a best fit has been performed for the interquark potential to the form $V(r)=V_{0}+a_{0} / r+\sigma r[12]$.

\section{$3 \quad$ Full QCD with $N_{f}=4$}

We have numerically simulated four flavours of quarks by using staggered fermions of bare mass $a m=0.05$. For the pure gauge sector of the theory the Wilson action has been chosen. The $\Phi$-type HMC algorithm [13] has been used for the updating. We have simulated at the following values of $\beta \equiv 6 / g^{2}=5.00,5.02,5.04,5.055 .06$ and 5.10 ( $g$ is the lattice bare coupling).

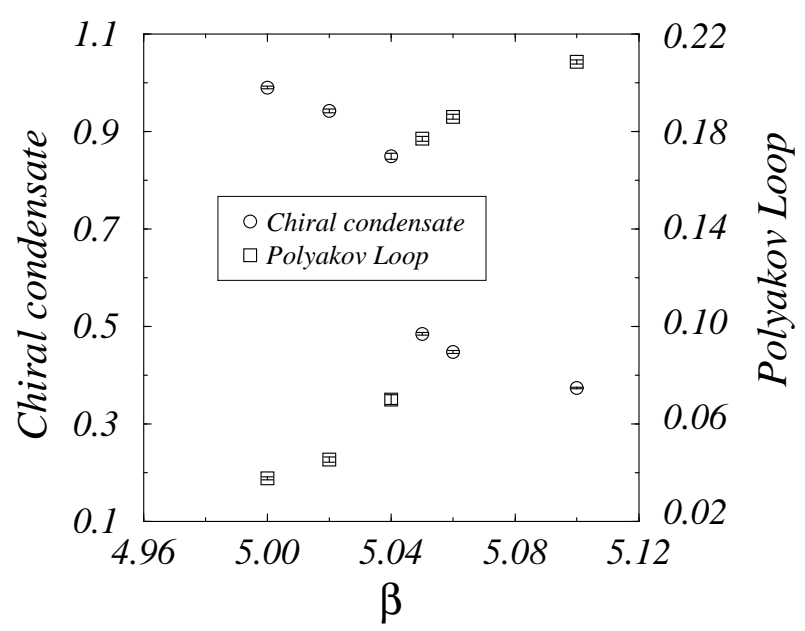

Figure 1: Polyakov loop and chiral condensate at finite $T$ for $N_{f}=4$.

The lattice volume was $16^{3} \times 4$. According to Ref. [14, at this temporal size the deconfinement transition occurs at $\beta_{c}=5.04$. We have checked this value by computing the Polyakov loop and the chiral condensate as shown in Fig. 11.

In the asymmetric lattice the temperature has been determined by the definition $T=1 /\left(a L_{t}\right)$, where $L_{t}$ is the temporal size.

We have checked that the distribution of topological charge is thermalized enough. In Fig. 2 we show such a distribution for $\beta=5.04$ after 30 cooling steps. At smaller values 
of $a m(a m \simeq 0.01)$ and higher $\beta$ 's, simulations are affected by a slowing down in the sampling [15, 16.

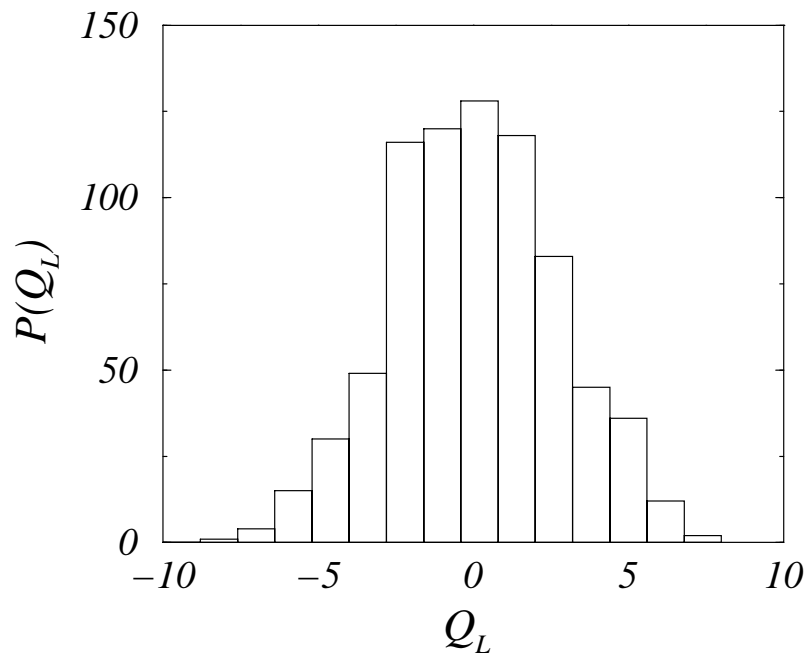

Figure 2: Distribution of topological charge $P\left(Q_{L}\right)$ at $\beta=5.04$.

In Table 1 we list the numerical results. The lattice spacing $a$ has been determined at $\beta=5.00,5.04$ and 5.10 on a $16^{4}$ lattice. For the other values of $\beta$, it was extrapolated by splines. From the value at $\beta_{c}, a=0.30(2) \mathrm{fm}$, we infer the critical temperature to be $T_{c}=164(11) \mathrm{MeV}$.

Table 1: $\chi, T / T_{c}$ and $a$ versus $\beta$ for $N_{f}=4$ on a $16^{3} \times 4$ lattice.

\begin{tabular}{cccl}
\hline \hline$\beta$ & $a / \mathrm{fm}$ & $T / T_{c}$ & $10^{-8} \times \chi / \mathrm{MeV}^{4}$ \\
\hline 5.00 & $0.31(4)$ & 0.9677 & $1.61(43)$ \\
5.02 & $0.306^{\dagger}$ & 0.9804 & $1.13(30)$ \\
5.04 & $0.30(2)$ & 1.0000 & $1.21(30)$ \\
5.05 & $0.296^{\dagger}$ & 1.0126 & $0.29(15)$ \\
5.06 & $0.292^{\dagger}$ & 1.0274 & $0.16(12)$ \\
5.10 & $0.270(8)$ & 1.1110 & $0.04(12)$ \\
\hline \hline
\end{tabular}

$\dagger$ Extrapolated, see text.

The behaviour of the ratio $\chi(T) / \chi(T=0)$ as a function of $T / T_{c}$ is shown in Fig. 3. The case $N_{f}=2$ will be discussed in the next section. For $N_{f}=4$ the value at zero temperature $\chi(T=0)$ is computed as the average of all results at $T \leq T_{c}$. For comparison, we have included in the figure the data for the quenched theory taken from Ref. [4]. There is clear evidence that the topological susceptibility in full QCD with $N_{f}=4$ presents a drop at $T_{c}$. Apparently the drop is sharper than in the case of pure Yang-Mills. However, the two theories have been simulated at rather different lattice volumes $\left(32^{3} \times 8\right.$ in the pure Yang-Mills case). Moreover, at the small values of $\beta$ used in our present simulation, we expect violations of scaling in the ratio $T / T_{c}$. As a consequence we prefer not to draw definite conclusions about the relative slopes. 


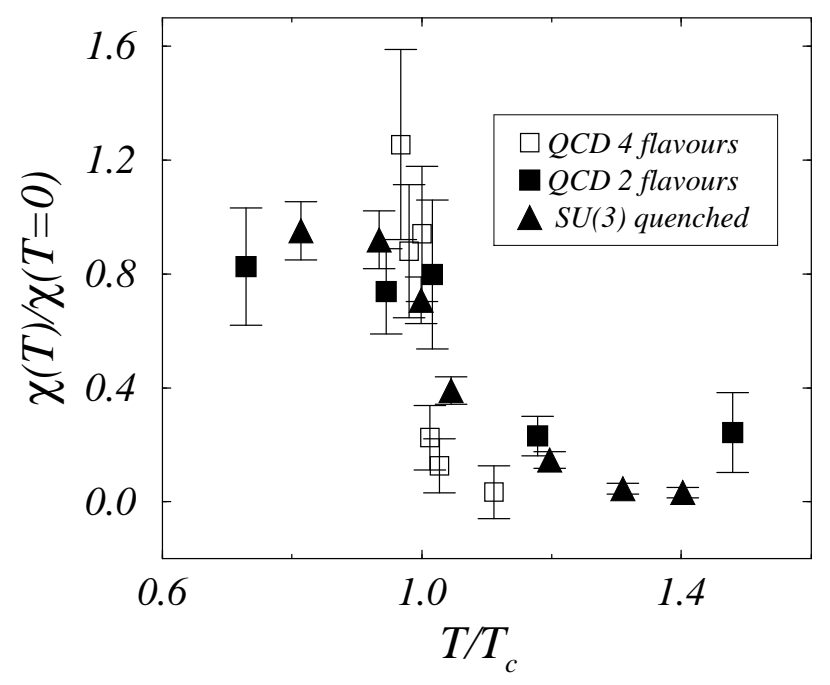

Figure 3: $\chi(T) / \chi(T=0)$ versus $T / T_{c}$ for $N_{f}=4, N_{f}=2$ and $N_{f}=0$.

\section{Full QCD with $N_{f}=2$}

Full QCD with two flavours of quarks has been simulated by using staggered fermions of bare mass $a m=0.0125$ and the usual Wilson action for the pure gauge sector. The updating has been performed with the R-type HMC algorithm [13], each trajectory consisting in 120 steps of $\Delta \tau=0.004$ units of molecular dynamics time. We have simulated at $\beta=5.40,5.50,5.55,5.60$ and 5.70 both at zero and finite temperature.

The lattice volume was $32^{3} \times 8$ for finite $T$ and $16^{4}$ for $T=0$. According to Ref. [17] at this temporal size the deconfinement transition occurs at $\beta_{c}=5.54(2)$. We have checked this number by studying the Polyakov loop in a similar way as in section 3 .

We have checked that the sets of configurations produced during the simulation are well decorrelated and the distribution of topological charge is well sampled. For the case of $\beta=5.70$ we needed a longer simulation to obtain a well sampled distribution of topological charge.

Table 2: $\chi, T / T_{c}$ and $a$ versus $\beta$ for $N_{f}=2$ on a $32^{3} \times 8$ lattice.

\begin{tabular}{cccl}
\hline \hline$\beta$ & $a / \mathrm{fm}$ & $T / T_{c}$ & $10^{-8} \times \chi / \mathrm{MeV}^{4}$ \\
\hline 5.40 & $0.1742(50)$ & 0.73 & $5.16(1.65)$ \\
5.50 & $0.1343(72)$ & 0.94 & $6.21(1.74)$ \\
5.55 & $0.1249(15)$ & 1.02 & $4.75(1.69)$ \\
5.60 & $0.1076(27)$ & 1.18 & $2.27(0.72)$ \\
5.70 & $0.0858(35)$ & 1.48 & $1.75(1.01)$ \\
\hline \hline
\end{tabular}

In Table 2 we report the data obtained from the simulation at finite $T$. From the value extrapolated at $\beta_{c}=5.54$, we infer the critical temperature to be $T_{c}=194(10)(15)$ 
$\mathrm{MeV}$ where the first error is our statistical and the second one the error induced by the indetermination in the value of $\beta_{c}$.

In Fig. [3 the behaviour of the ratio $\chi(T) / \chi(T=0)$ as a function of $T / T_{c}$ is shown. For $N_{f}=2$ the value at zero temperature $\chi(T=0)$ has been obtained from an independent simulation on symmetric lattices $16^{4}$ at the same values of $\beta$ (see Table 3). There is a clear drop in the signal when the deconfinement temperature is crossed.

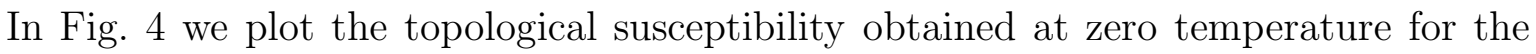
five values of $\beta$. The corresponding set of data is listed in Table 3. Having fixed $a m$, the values of the bare mass vary as shown in the upper horizontal scale. A fit with a constant value of the topological susceptibility gives $\chi=(163 \pm 6)^{4} \mathrm{MeV}^{4}$, with the statistical test (chi-square)/d.o.f $=0.37$; a fit with a linear homogeneous dependence on $m$, like Eq. (3), gives $\langle\bar{\psi} \psi\rangle_{\text {BARE }}=(6.2 \pm 0.8) \times 10^{7} \mathrm{MeV}^{3}$ and (chi-square)/d.o.f $=0.94$. Therefore our present data are compatible with both behaviours, and we are not able, within our errors, to check Eq. (3).

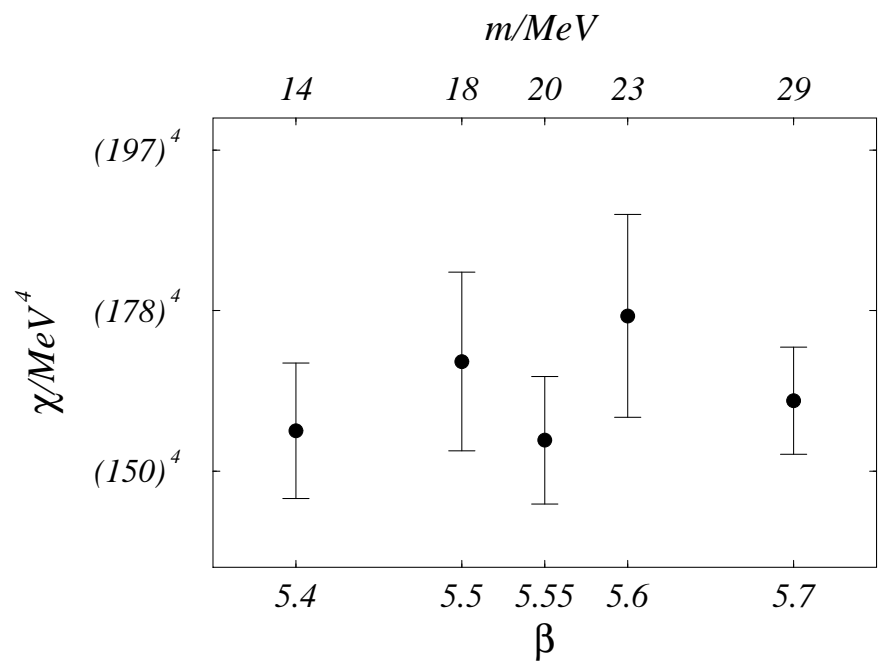

Figure 4: Dependence of the topological susceptibility on the bare quark mass for $N_{f}=2$ at zero temperature.

Table 3: $\chi$ versus $\beta$ for $N_{f}=2$ on a $16^{4}$ lattice.

\begin{tabular}{cl}
\hline \hline$\beta$ & $10^{-8} \times \chi / \mathrm{MeV}^{4}$ \\
\hline 5.40 & $6.25(2.11)$ \\
5.50 & $8.41(2.78)$ \\
5.55 & $5.96(1.99)$ \\
5.60 & $9.83(3.16)$ \\
5.70 & $7.19(1.67)$ \\
\hline \hline
\end{tabular}




\section{Conclusions}

We have studied the topological susceptibility $\chi$ in full QCD with 2 and 4 flavours of dynamical fermions. At zero temperature, we have not enough precision to check the chiral limit, Eq. (3).

At finite temperature, $\chi$ stays constant for values of $T$ up to the deconfinement temperature $T_{c}$. At $T_{c}$ it presents a sudden drop and $\chi$ becomes compatible with zero at

$T \simeq 1.5 T_{c}$ for $N_{f}=2$ and $T \simeq 1.2 T_{c}$ for $N_{f}=4$. This behaviour is qualitatively similar to that found in Ref. [䧃] for the quenched case.

\section{Acknowledgements}

B.A. thanks the Theory Group in Pisa for the kind hospitality. Financial support from EC, Contract FMRX-CT97-0122, and from MURST is acknowledged. 


\section{References}

[1] E. Witten, Nucl. Phys. B156 (1979) 269.

[2] G. Veneziano, Nucl. Phys. B159 (1979) 213.

[3] R. J. Crewther, Riv. Nuovo Cim. 2, No 8 (1979) 63.

[4] B. Allés, M. D’Elia, A. Di Giacomo, Nucl. Phys. B494 (1997) 281.

[5] M. Teper, plenary talk at the Lattice-99 conference, hep-lat/9909124.

[6] F. C. Hansen, H. Leutwyler, Nucl. Phys. B350 (1991) 201.

[7] T. Schäfer, E. V. Shuryak, Rev. Mod. Phys. 70 (1998) 323.

[8] M. Campostrini, A. Di Giacomo, H. Panagopoulos, Phys. Lett. B212 (1988) 206.

[9] B. Allés, A. Di Giacomo, H. Panagopoulos, E. Vicari, Phys. Lett. B350 (1995) 70.

[10] C. Christou, A. Di Giacomo, H. Panagopoulos, E. Vicari, Phys. Rev. D53 (1996) 2619.

[11] A. Di Giacomo, E. Vicari, Phys. Lett. B275 (1992) 429.

[12] G. S. Bali, K. Schilling, Phys. Rev. D46 (1992) 2636.

[13] S. Gottlieb, W. Liu, D. Toussaint, R. L. Renken, R. L. Sugar, Phys. Rev. D35 (1987) 2531.

[14] F. R. Brown, F. P. Butler, H. Chen, N. H. Christ, Z.-H. Dong, W. Schaffer, L. I. Unger, A. Vaccarino, Phys. Lett. B251 (1990) 181.

[15] B. Allés, G. Boyd, M. D’Elia, A. Di Giacomo, E. Vicari, Phys. Lett. B389 (1996) 107.

[16] B. Allés, G. Bali, M. D’Elia, A. Di Giacomo, N. Eicker, K. Schilling, A. Spitz, S. Güsken, H. Hoeber, Th. Lippert, T. Struckmann, P. Ueberholz, J. Viehoff, Phys. Rev. D58 (1998), 071503.

[17] S. Gottlieb, A. Krasnitz, U. M. Heller, A. D. Kennedy, J. B. Kogut, R. L. Renken, D. K. Sinclair, R. L. Sugar, D. Toussaint, K. C. Wang, Phys. Rev. D47 (1993) 3619. 